\title{
СПОСОБЫ И МЕТОДЫ ПОВЫШЕНИЯ ЭФФЕКТИВНОСТИ МЕЛКОСЕРИЙНОГО ПРОИЗВОДСТВА
}

\section{WAYS AND METHODS FOR IMPROVING THE EFFICIENCY OF SMALL-SCALE PRODUCTION}

S. Savinov

Summary. The methods and methods of increasing the efficiency of smallscale production, the features of the structure of the organization of the technological process of production are considered.

Keywords: operational and calendar planning, small-scale production, production efficiency, production management system.
Савинов Сергей Владимирович

Соискатель, ФГБОУВО «Владимирский государственный университет имени Александра Григорьевича и Николая Григорьевича Столетовых» conf-mail@bk.ru

Аннотация. Рассматриваются способы и методы повышения эффективности мелкосерийного производства, особенности структуры организации технологического процесса производства.

Ключевые слова: оперативно-календарное планирование, мелкосерийное производство, эффективность производства, система управления производством.

сом является создание максимально эффективного оперативно-календарного производственного плана использования технологического оборудования и работающего на предприятие персонала. Данная задача реализуется с помощью применения специализированных алгоритмов планирования технологического процесса, а именно организации максимальной степени загрузки производственных мощностей и обеспечения оперативного взаимодействия между ключевыми производственными органами и службами по управлению производством. Для обеспечения высокой эффективности производственной деятельности предприятия целесообразно рекомендовать обязательное внедрение автоматизированных систем управления производством.

Для обеспечения возможности оперативного управления и планирования производственным процессом активно внедряются системы на базе: MRP / MRP_II (Material Requirements Planning - планирование материальных ресурсов); ERP (Enterprise Resource Planning - планирование ресурсов предприятия); MES (Manufacturing Execution System - автоматизированная исполнительная система производственного уровня); APS (Advanced Planning and Scheduling - усовершенствованное планирование).

Если рассмотреть процесс управления применительно к сложным многоуровневым производственным системам, то происходит последовательная обработка и передача информации с нижнего уровня на верхние уровни производственной структуры, формируя при этом корпоративную информационную систему (КИС). На первом этапе внедрения и функционирования КИС
Целевой задачей использования автоматизированной системы управления производственным процес- 


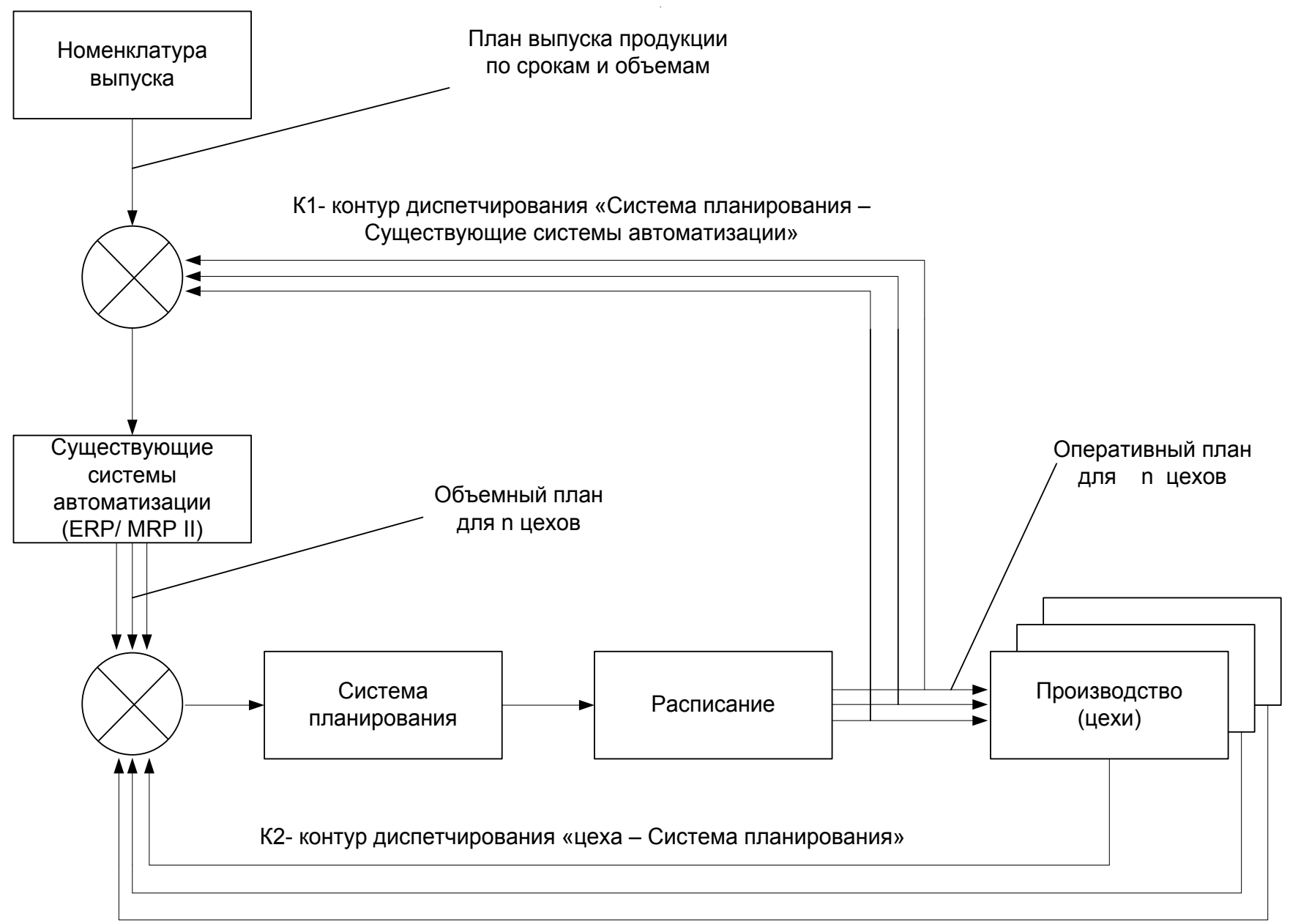

Рис. 1. Двухзвенная схема системы планирования на производстве.

необходимо обеспечить внедрение инструментариев информатизации в приоритетные службы для организации начала технологического процесса и обеспечения связи между производственными уровнями. Сформированные производственные информационные системы в соответствии с моделью СІМ (Computer-Integrated Manufacturing - комплексная автоматизация производства) должны быть разделены, учитывая два основных параметра: степень дискретности временной производственной оси; количество обрабатываемых данных, формируемых в процессе производственной деятельности на образованных уровнях.

Совокупность информационных систем складывается в специфическую концептуальную пирамиду, в которой происходит иерархическое взаимодействие между уровнями. Нижний уровень включает в себя технологические системы управления на рабочих местах. Второй уровень включает в себя системы управления повторяющимися технологическими процессами. Верхний - третий уровень взаимодействуют с MES-система- ми, WMS-системами, APS-системами. Четвертый уровень представлен ERP-системами. В качестве дополнительного базового уровня можно выделить нулевой уровень с технологическим оборудованием. Управление всеми уровнями сформированной СІМ-пирамиды обеспечивается по средствам внедрения OLAP - системы для функционирования бизнес-аналитических процессов, благодаря чему появляется возможность сбора и обработки большого количества данных из нижерасположенных ERP-систем, для формирования необходимых корректирующих мер по изменению оперативно-календарного производственного плана предприятия.

Модель СІМ позволила создать промышленный стандарт предприятий ISA 95, внедрение которого позволило установить особенности в разделении между системами управления, сконцентрированными на финансово-хозяйственной деятельности и непосредственно информационными системами организации технологическими производственными службами предприятия. Рассмотрим процесс создания и функционирования 


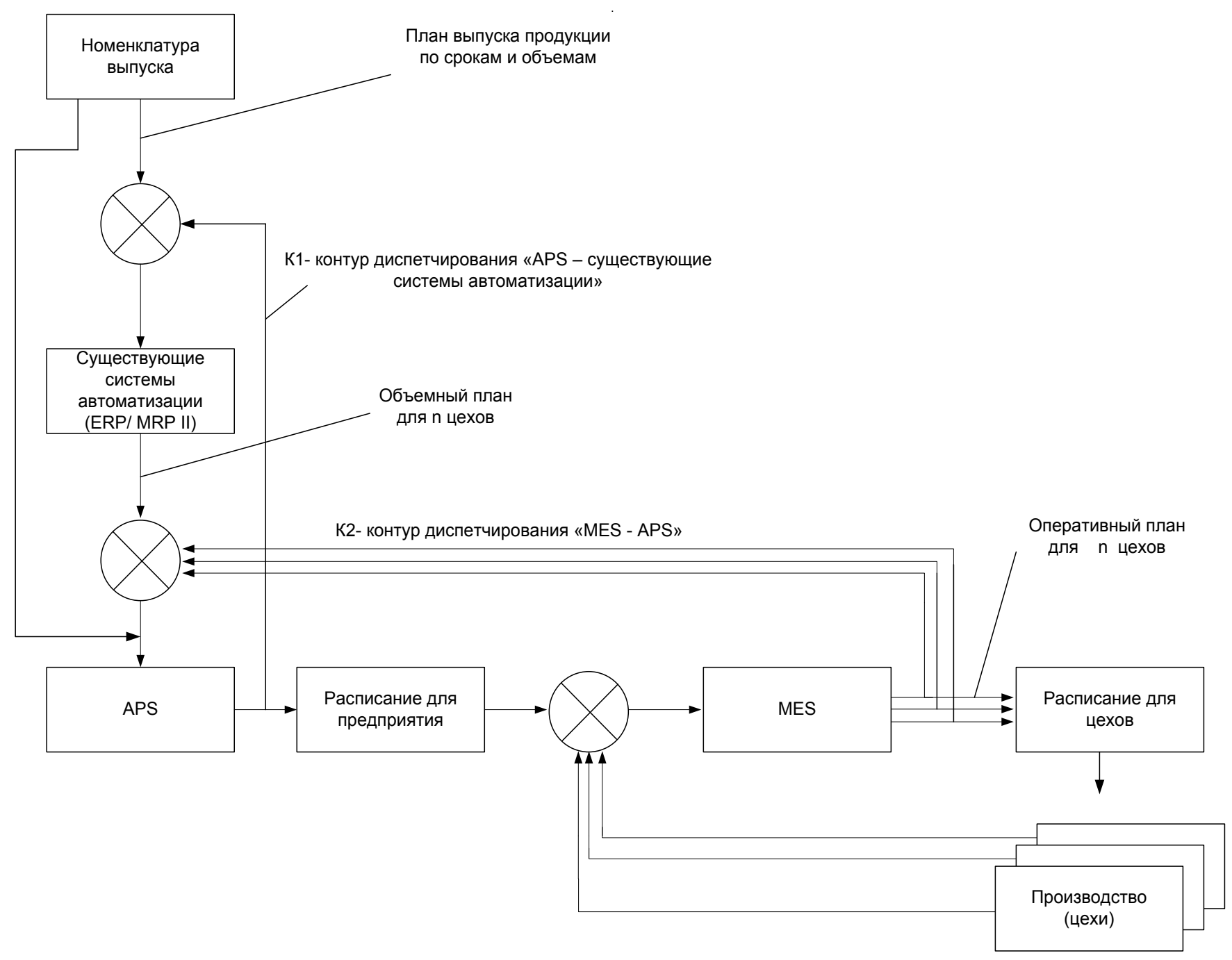

Рис. 2. Трехзвенная схема системы планирования на производстве.

корпоративной информационной системы на предприятии, состоящий из нескольких идущих последовательно за собой этапов автоматизации.

Базовый или первый этап обеспечивается за счет использования автоматизации различного рода финансово-денежных документов, что значительно повышает эффективность, скорость обработки, использования финансовых ресурсов. Базовый этап внедряется повсеместно, в том числе для облегчения работы самого предприятия при взаимодействии с государственными службами и т.п.

Следующий этап является более затратным и емким по времени внедрения - использование в производстве компьютерных средств автоматизированного проектирования Computer-Aided Design (CAD), а также средств автоматизации инженерных расчетов Computer-Aided Engineering (CAE). Благодаря активно- му внедрению и использованию CAD- и CAE- систем удается создать электронную версию базы продукции предприятия. В качестве этапа автоматизации непосредственно для технологического производственного оборудования - CAM (Computer-Aided Manufacturing).

На завершающем этапе обеспечивается включение в автоматизированную систему комплекс PDM/PLM систем, благодаря чему обеспечивается возможность организации единой области информационного пространства для данных о технологической подготовке производства для номенклатуры изделия. Системы, относящиеся к классу APS и MES, уже производят оценку эффективности организации оперативно-календарного планирования и при необходимости произвести коррективы для увеличения степени загрузки технологических станций при производстве продукции. 
Конструкторская, нормативная документация, директивные сроки изготовления и т.п.

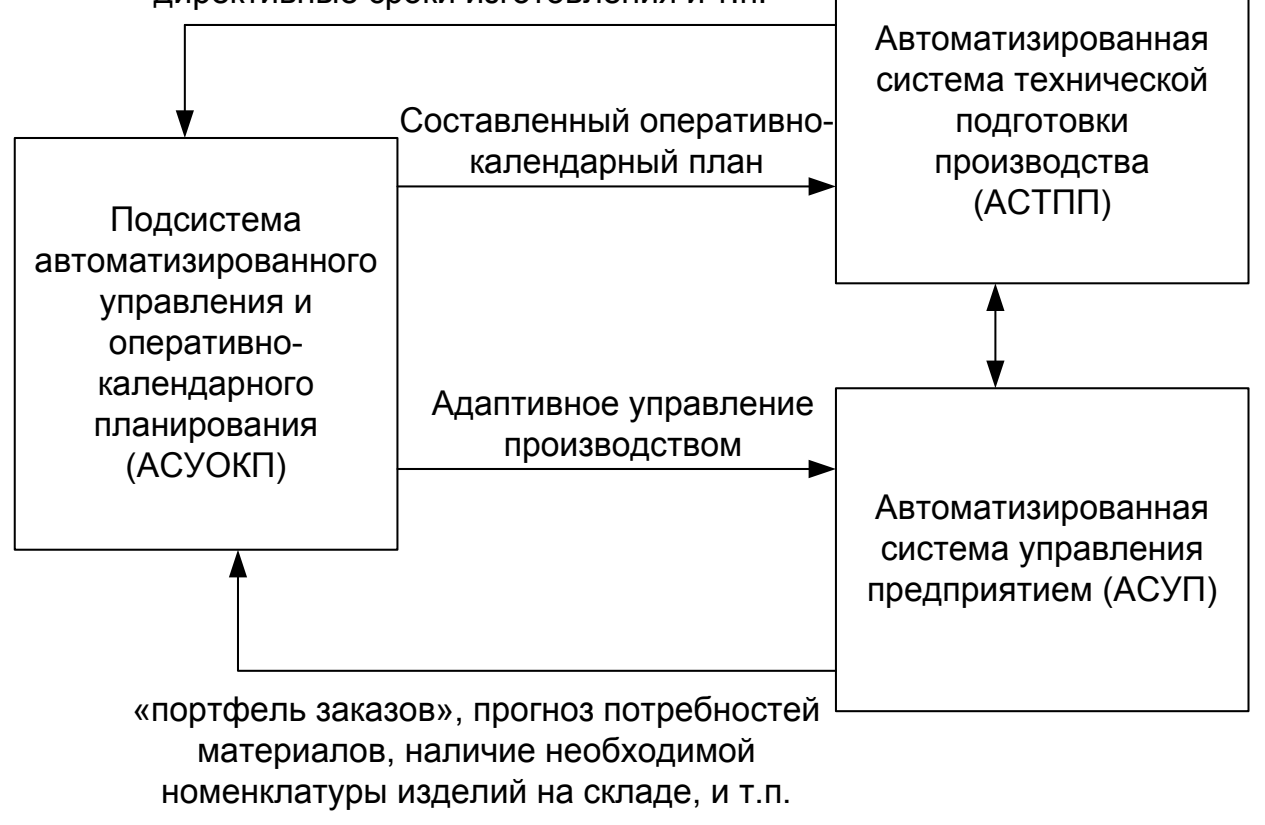

Рис. 3. Функциональная связь между интегрированной подсистемой управления и оперативнокалендарного планирования и АСТПП, АСУП.

В качестве используемой системы управления на предприятиях мелкосерийного производства как правило используются две основных схемы организации планирования: двухзвенная и трехзвенная.

Двухзвенная схема системы планирования в своей основе использует совместное использование системы планирования предприятия и ERP/MRP II систем.

Трехзвенная схема системы планирования в отличие от двухзвенной системы планирования использует сочетание интегрированных автоматизированных систем предприятия и APS и MES систем.

Для разработки высокоэффективной системы оперативного управления планированием на производстве должна быть сформирована соответствующая математическая модель, учитывающая не только факторы технологических процессов производства, но и возможные варианты возникновения проблем на производстве.

На первом этапе происходит формирование технического задания на необходимый тип выпускаемой продукции. Затем соответствующие структурные подразделения предприятия разрабатывают комплект конструкторской документации либо используется ранее разработанная или приобретенная документация на изделие. Наличие соответствующего комплекта документации позволит обеспечить технологическую подготовку производственного процесса, определив при этом необходимый перечень технологических операций и тип используемого оборудования. Имея информацию о технологической подготовке производства можно непосредственно переходить к формирования оперативного-календарного планирования как для технологических процессов, так и всего производственного процесса. Оперативно-календарное планирование формируется в автоматизированной системе управления производственным процессом, а именно в отдельно-выделенной подсистеме для расчета и составления временных затрат на производство.

Разработанный модуль подсистемы автоматизированного управления и оперативно-календарного планирования (АСУОКП) постоянно взаимодействует с автоматизированной системой технической подготовки производства (АСТПП) и автоматизированной системой управления предприятием (АСУП). Информационная структура подсистемы как правило представляется в виде множества графов для каждого изделия, которые формирует в совокупности единый граф производственного процесса, который необходимо оптимизировать и вносить коррективы для оперативной реакции на возникновение факторов производства, не включенных в ранее сформированный оперативный план.

Продукция в информационной структуре подсистемы АСУОКП представляется в виде графа - дерева, 


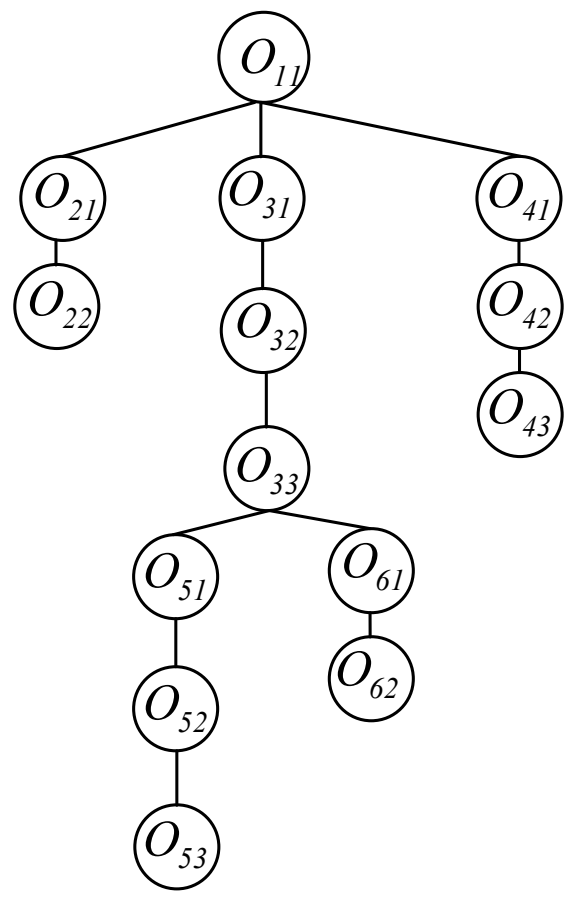

Рис. 4. Граф изделия

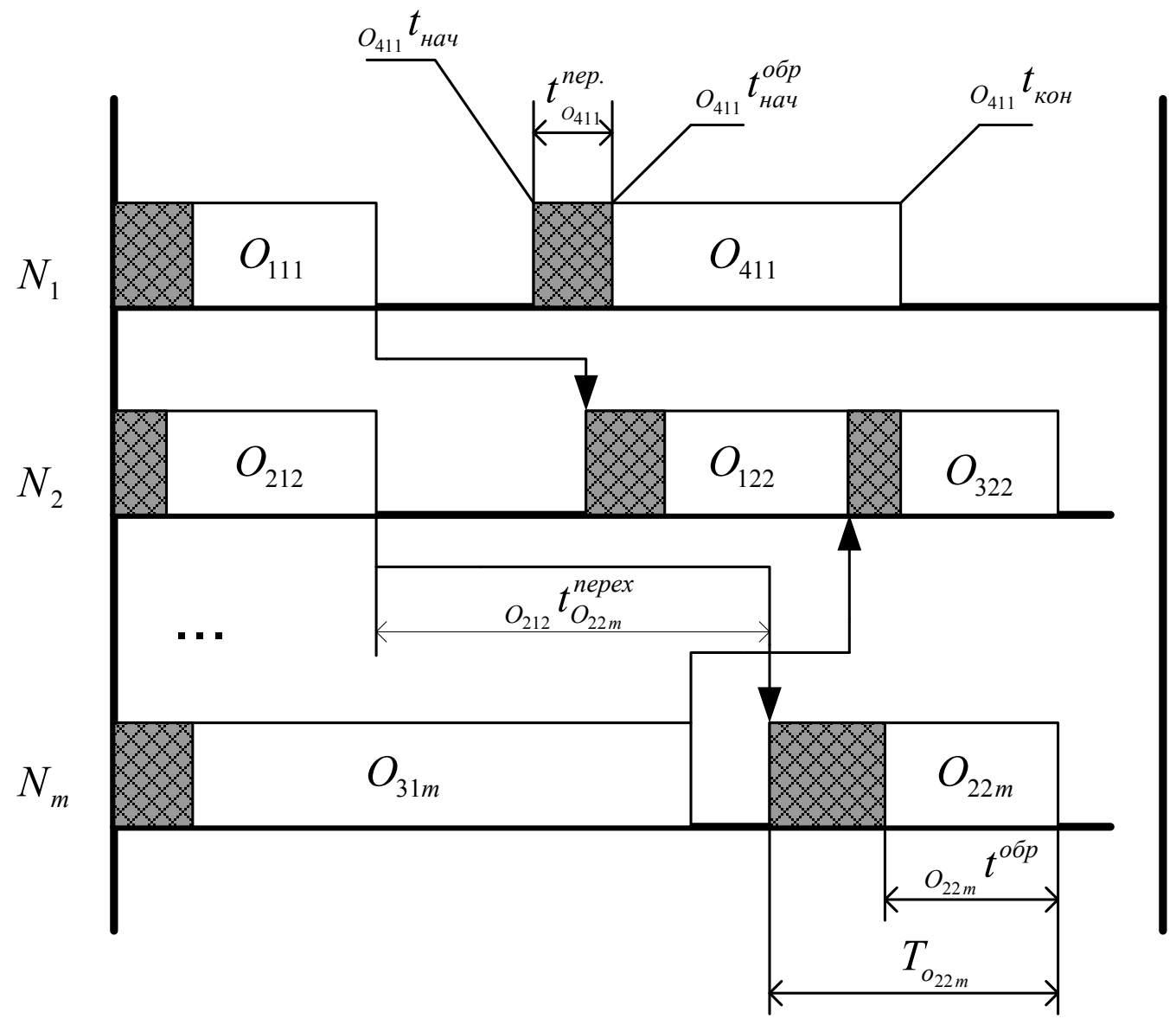

Рис. 5. Фрагмент технологического расписания. 
который состоит из вершин, являющимися основными производственными сборочными единицами. Ветви графа соединяют вершины в необходимой последовательности выполнения технологических операций.

Граф продукции формирует технологические маршруты для каждой составной части производимой продукции. На технологических маршрутах формируется необходимый перечень технологических операций $\left(O_{i}\right.$, $j)$, длина маршрута зависит от количества операций которые указаны в конструкторской документации. Технологическая операция имеет соответствующий временной параметр - время выполнения, на основании чего формируется расписание технологических маршрутов.

На рисунке 5 представлен фрагмент технологического расписания, в котором учитываются временные параметры: время начало операции $t_{\text {нач}}$ время окончания операции $t_{\text {кон }}$ (Время выполнения технологической операции $\left.T_{i, j}=t_{i-1, j-1}^{\text {нач }}+t_{i, j}^{\text {кон }}\right)$. Кроме выполнения основных технологических операций, существуют также вспомогательные технологические операции - время переналадки (перепрограммирования) технологической станции $t^{\text {nep }} O_{i, j}$ для обеспечения возможности перенастройки технологической станции на выполнение другой операции или изменения конфигурации технологической оснастки. Время необходимое для передачи изделия между технологическими станциями - время переходной транспортной операции $O_{i-1, j-1} t^{\text {nepex }} O_{i, j}$. Совокупность длительностей всех технологических маршрутов $N_{l} \cdot N_{m}$ образует ветки графа информационной подсистемы. Проведение оптимизации полученного графа позволяет получить эффективный оперативно-календарный план, позволяющий обеспечить максимальное значение загруженности технологических станций и снижение времени простоя оборудования даже при мелкосерийном производстве.

При расчете оптимального технологического расписания необходимо обрабатывать два условия: при организации производства продукции, состоящей из изделий с большим количеством технологических операций, увеличивается вероятность возникновения нехватки материалов и составных частей, т.к. образуется партия изделий, которая имеет большие временные задержки в технологическом расписании, а следовательно, очень сложно оперативно обеспечить все технологические станции необходимым количеством материалов для производства; если наоборот продукция изготавливает- ся путем выполнения небольшого количества технологических операций, то в этом случае значительно возрастает время переналадки (перепрограммирования) технологических станций для выполнения нового типа производственной операции, это конечно снижает степень загруженности технологического оборудования, но уменьшает показатели эффективности.

Поэтому для организации эффективного календарного планирования необходимо на основании типа изготавливаемой продукции определить оптимальную степень загрузки технологических станций - в случае увеличении длины партии изделий необходимо произвести принудительное разделение этапов технологического процесса на несколько вспомогательных; - в случае уменьшения длины партии изделий необходимо выполнить группировку смежных технологических операций из нескольких технологических расписаний видов продукции. Кроме того, в технологическое расписание должны быть заложены временные задержки на обработку внештатных ситуаций, таких как выход из строя оборудования на технологической станции, изменение характеристик применяемых в производстве материалов, что приводит к изменению времени обработки единицы продукции. Частоту появления отказов оборудования можно значительно уменьшить, включив в расчеты календарного планирования время на проведение технологического обслуживания (ТО) как планового, так и непланового, при этом ТО технологических станций можно производить без остановки производства, необходимо при составлении расписания производимых работ закладывать ТО на тех технологических станциях, которые производят минимальное количество операций или имеют максимальное время простоя между операциями технологического процесса.

Таким образом для того, чтобы повысить показатели эффективности мелкосерийного производства необходимо произвести равномерное распределение нагрузки по технологическим станциям, учитывая при этом возможность перенаправления потоков деталей в процессе производства; сформировать технологическое расписание с минимальными возможными временными задержками. Отдельно стоит учитывать факторы возникновения отказов оборудования и необходимость проведения ТО технологических станций. Грамотное использование средств для создания оперативно-календарных планов позволяет в значительной мере повысить эффективность производства.

\section{ЛИТЕРАТУРА}

1. Антонов А.М. Основы современной организации производства / А.М. Антонов. - М.: ИНФРА-М., 2004. - 432 с.

2. Зориктуев В.Ц., Загидуллин Р.Р. Оперативно-календарное планирование в гибких производственных системах. Учебное пособие. Изд.УГАТУ,- Уфа, 2004. $-106 \mathrm{c}$ 
3. Бухалков М.И. Внутрифирменное планирование: Учебник. - М.: Инфра- М., 1999.-392с.

4. Kalpakjian S. Manufacturing engineering and technology / Kalpakjian Serope, Schmid Steven // Prentice Hall (5th ed.), — 2006, — p.1192

5. Коноплев А.Н. Разработка функциональной модели для системы оперативно-производственного планирования /Коноплев А.Н.// Материалы XVIII Международной конференции по вычислительной механике и современным прикладным программным системам (ВМСППС»2013), 22-31 мая 2013 г., Алушта. — М.: Изд-во МАИ, 2013-888с. - C. 769-771

6. ЦЦарев В.В. Внутрифирменное планирование.- СПб.: Питер, 2002.-496 с.

7. Сочнев А.Н. Оперативное управление производственными системами на основе сетей Петри диссертация ... кандидата технических наук: 05.13 .01 Красноярск, 2005-153 с.

8. Омельченко И.Н. Промышленная логистика. Логистико-ориентированное управление организационно-экономической устойчивостью промышленных предприятий в рыночной среде / И.Н. Омельченко, А.А. Колобов, А.Ю. Ермаков, А.В. Киреев; Под ред. А.А. Колобова.— М.: МГТУ им. Н.Э. Баумана, 1997.

9. Дотзабаев К.Т. Производственный менеджмент. Учебное пособие. / К.Т. Дотзабаев, А.Т. Гритин, Г.К. Джурабаева.— М.: Кнорус, 2005.

(c) Савинов Сергей Владимирович ( conf-mail@bk.ru ).

Журнал «Современная наука: актуальные проблемы теории и практики»

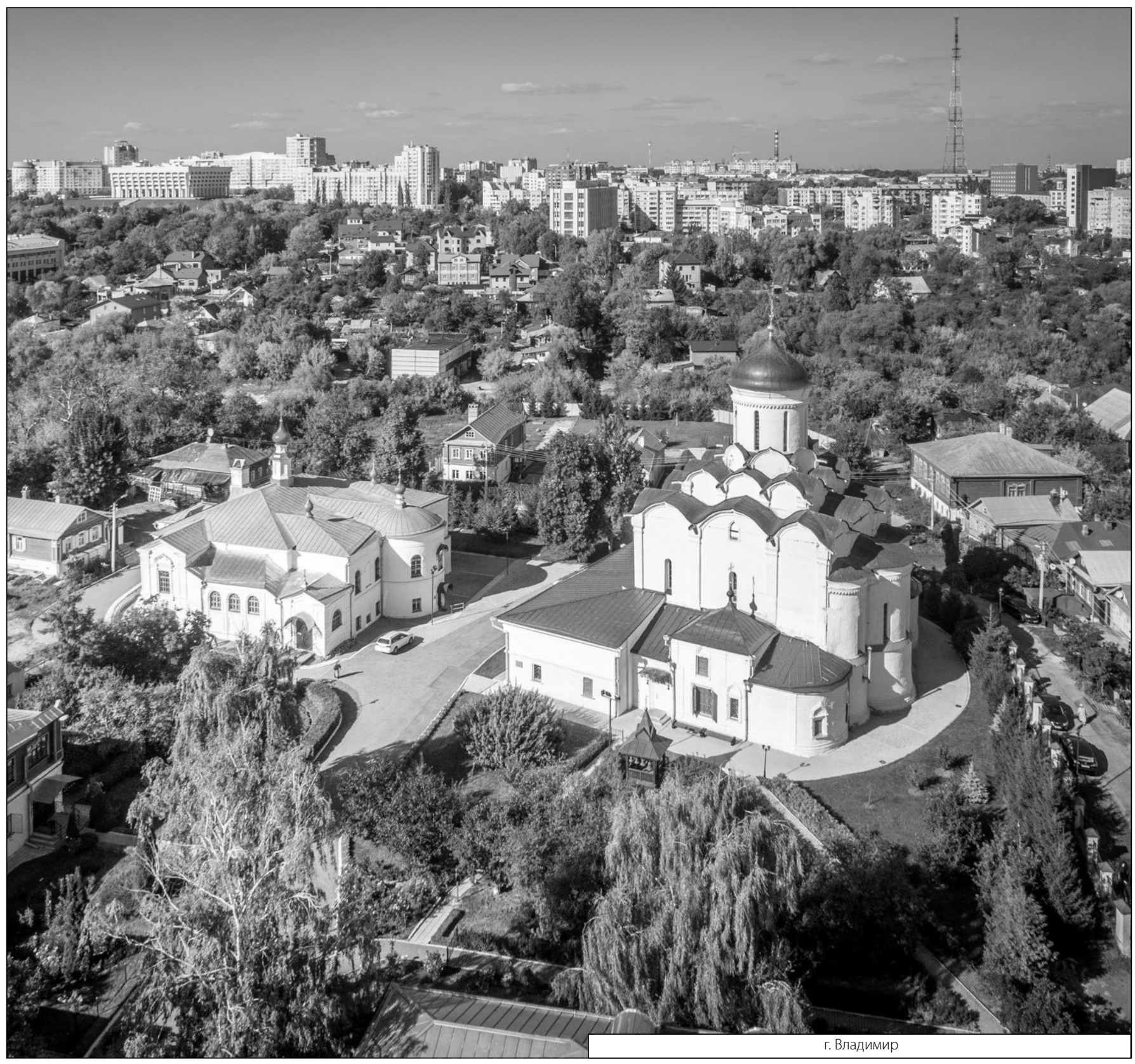

